\title{
Microglia complement astrocytes in neuromyelitis optica
}

\author{
Zahra Moinfar and Scott S. Zamvil \\ Department of Neurology, Weill Institute of Neurosciences and Program in Immunology, UCSF, San Francisco, California, USA
}

\begin{abstract}
Neuromyelitis optica (NMO) is a central nervous system (CNS) inflammatory autoimmune disease caused by antibodies against aquaporin-4 (AQP4) expressed on astrocytes. Binding of AQP4-specific antibodies (NMO-IgC) triggers activation of the complement cascade, which is responsible for astrocyte loss and secondary demyelination. Although the role for the cytolytic complement proteins in astrocyte destruction in NMO is well established, little is known regarding the initial phase of astrocyte injury. In this issue of the $J C l$, Chen and colleagues evaluated the precytolytic phase when NMO-IgC binds astrocytes in vivo in the absence of exogenous complement. NMO-IgC alone caused astrocyte activation and AQP4 loss. Surprisingly, microglia, CNS-resident innate immune cells that produce endogenous complement, were required for clinical manifestations of disease, a finding that suggests microglia may serve as a therapeutic target in NMO.
\end{abstract}

\section{Complement mediates tissue destruction in NMO}

Neuromyelitis optica (NMO) is a rare, disabling, sometimes fatal, central nervous system (CNS) autoimmune disease that is characterized by severe attacks of visual loss and paralysis. NMO is caused by antibodies that target the water channel protein aquaporin-4 (AQP4) concentrated on astrocytic foot processes at the blood-brain barrier $(1,2)$. The AQP4specific antibodies (NMO-IgG) are IgG1, a $\mathrm{T}$ cell-dependent antibody subclass, emphasizing cooperation between cellular and humoral arms of adaptive immunity in NMO pathogenesis (3). IgG1 is an antibody subclass that is capable of activating complement. It is generally accepted that the terminal cytolytic complement proteins have a central role in CNS infiltration of neutrophils, macrophages, and eosinophils, as well as astrocyte destruction and secondary demyelination in NMO (4, 5). Thus, NMO is considered a complement-mediated astrocytopathy (5). However, the initial stage of astrocyte injury after binding of NMO-IgG to astrocytes is not well understood.

\section{NMO-IgG-induced astrocytic C3 production promotes microglial activation}

In the current issue of the JCI, Chen and his colleagues examined the role of NMO-

\section{Related Article: p. 4025}

Conflict of interest: SSZ is Deputy Editor of Neurology and Neuroimmunology and Neuroinflammation and is an Associate Editor for Frontiers in Immunology and Frontiers in Neurology. He serves on the Advisory Committee for the American Congress on Treatment and Research in Multiple Sclerosis (ACTRIMS) and is a standing member of the research grant review committee for the National Multiple Sclerosis Society (NMSS). He has served on the Editorial Board of the Journal of Clinical Investigation, Journal of Immunology, and Journal of Neurological Sciences, and has been a charter member of the grant review committee for the NIH Clinical Neuroimmunology and Brain Tumors (CNBT) Study Section. He has served or serves as a consultant and received honoraria from Alexion, Biogen-Idec, EMD-Serono, Genzyme, Novartis, Roche/Genentech, and Teva Pharmaceuticals Inc., and has served on Data Safety Monitoring Boards for Lilly, BioMS, Teva, and Opexa Therapeutics.

Copyright: () 2020, American Society for Clinical Investigation.

Reference information: / Clin Invest. 2020;130(8):3961-3964. https://doi.org/10.1172/JCI138804.

IgG in the early stage of CNS tissue injury using a mouse model (6). When WT mice were subjected to continuous intrathecal infusion of NMO-IgG, recipient mice developed progressive hind-limb paralysis that was partially reversible upon discontinuation of NMO-IgG. Motor impairment was AQP4 specific, as it was not observed when WT mice were given control IgG or when $\mathrm{AQP} 4$-specific IgG was administered to AQP4-deficient mice. As in NMO, administration of NMO-IgG to WT mice was associated with loss of AQP4 expression on astrocytes. However, astrocytes remained viable and there was no evidence of terminal complement activation. Further, there was both prominent activation and proliferation of microglia. To investigate how microglia may participate in this disease process, microglia were selectively depleted transiently in vivo. In this model, administration of NMO-IgG caused astrocytic AQP4 loss independently of the presence or absence of microglia. NMO-IgG-induced paralysis did not correspond to the time of maximal microglial depletion. Rather, motor impairment in NMO-IgG-treated mice did not occur until microglia were replenished. Confocal imaging revealed an abundance of activated microglia with substantial spatial overlap with astrocytes, and 2-photon imaging demonstrated convergence of microglial processes toward astrocytes. Thus, the authors questioned whether microglia and astrocytes cooperate in NMO lesion development. In this regard, it is known that NMO-IgG can elicit significant production of C3 by astrocytes (7) and in addition, microglia express C3a receptor (C3aR) (8). Thus, astrocytes may promote microglial activation via the $\mathrm{C} 3$ cleavage product, C3a. Indeed, NMO-IgG infusion was associated with marked upregulation of C3 expression in astrocytes and C3aR on microglia (Figure 1A). Microgliaastrocyte spatial overlap and motor impairment were not observed when NMO-IgG was administered to C3aR-deficient mice, 
A

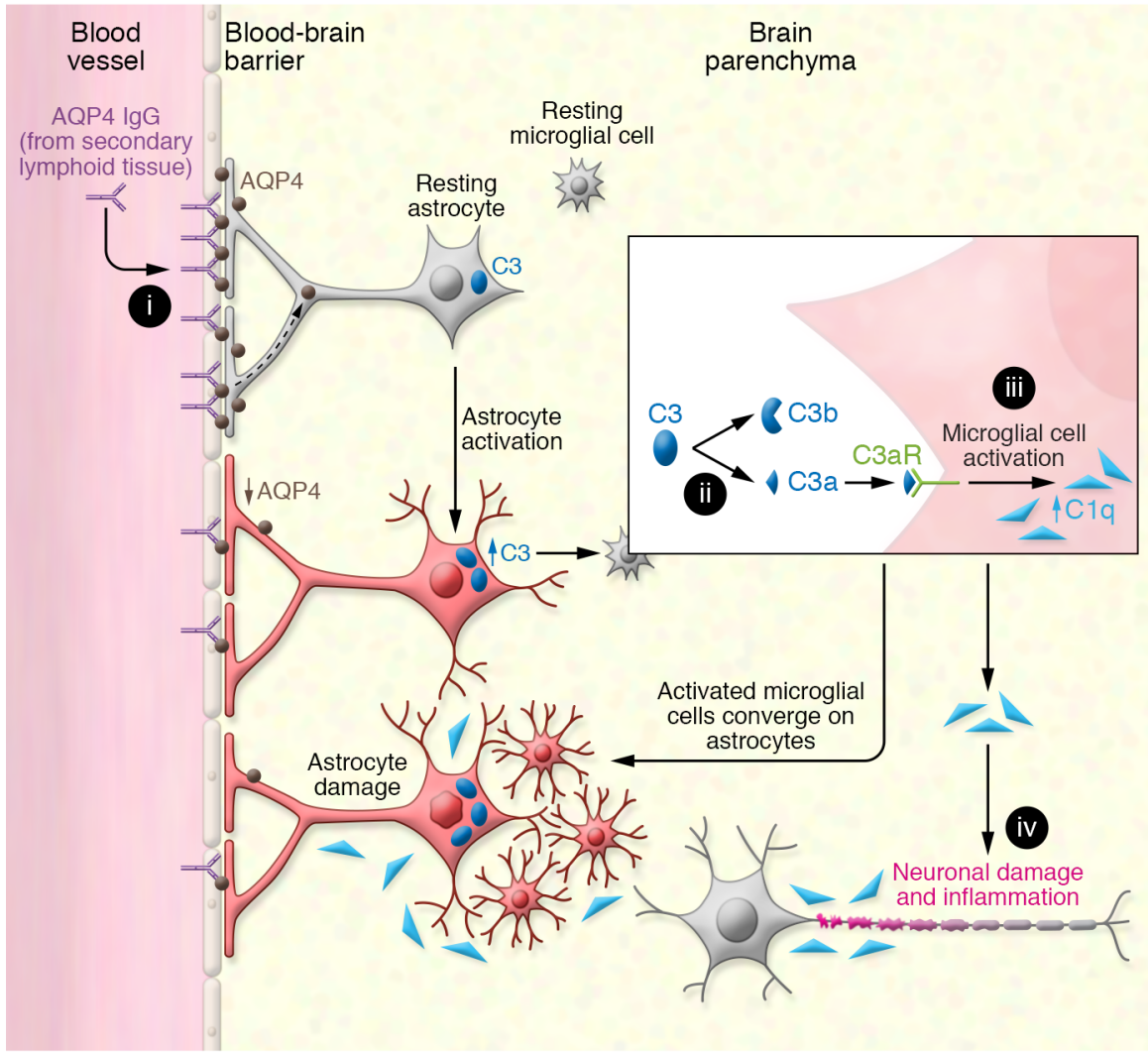

Figure 1. Astrocytes cooperate with microglia in complement-mediated CNS damage in NMO. (A) A model for astrocyte-microglia interaction in precytolytic tissue injury in NMO. (i) AQP4-specific IgG1 is produced in secondary lymphoid tissue outside the CNS. AQP4-specific antibodies bind to AQP4 on astrocytic endfoot processes, leading to AQP4 internalization, reduced cell surface AQP4 expression, and astrocytic activation. (ii) Activated astrocytes produce elevated C3 that is cleaved to C3a and C3b. (iii) Secreted C3a binds C3aR on resting microglia, promoting microglial activation, production of $\mathrm{C} \mathrm{q}$, and convergence toward astrocytes. (iv) C1q promotes localized injury to neurons and oligodendrocytes. (B) Activation of the classical complement cascade leads to sustained CNS tissue damage in NMO. (i) C1q binds the framework constant $(\mathrm{Fc})$ region of adjacent AQP4 antibodies bound to AQP4 in close proximity, attracting $\mathrm{C} 1 \mathrm{r}$ and $\mathrm{C} 1 \mathrm{~s}$. (ii) This $\mathrm{C} 1$ complex activates $\mathrm{C} 4$ and $\mathrm{C} 2$ components that lead to formation of C3 convertase, producing $C 3 \mathrm{~b}$, which together with $\mathrm{C} 4 \mathrm{~b}$ and $\mathrm{C} 2 \mathrm{~b}$ create the $C 5$ convertase. Eculizumab (Soliris), a C5-convertase inhibitor, is approved for treatment of NMO. (iii) $C 5 b$, one of the 2 C5 cleavage products, together with $\mathrm{C6}-\mathrm{C} 9$ proteins form the membrane attack complex (MAC), which leads to astrocyte lysis and tissue necrosis. This figure was adapted with permission from Kenneth Probst (Xavier Studio).

B

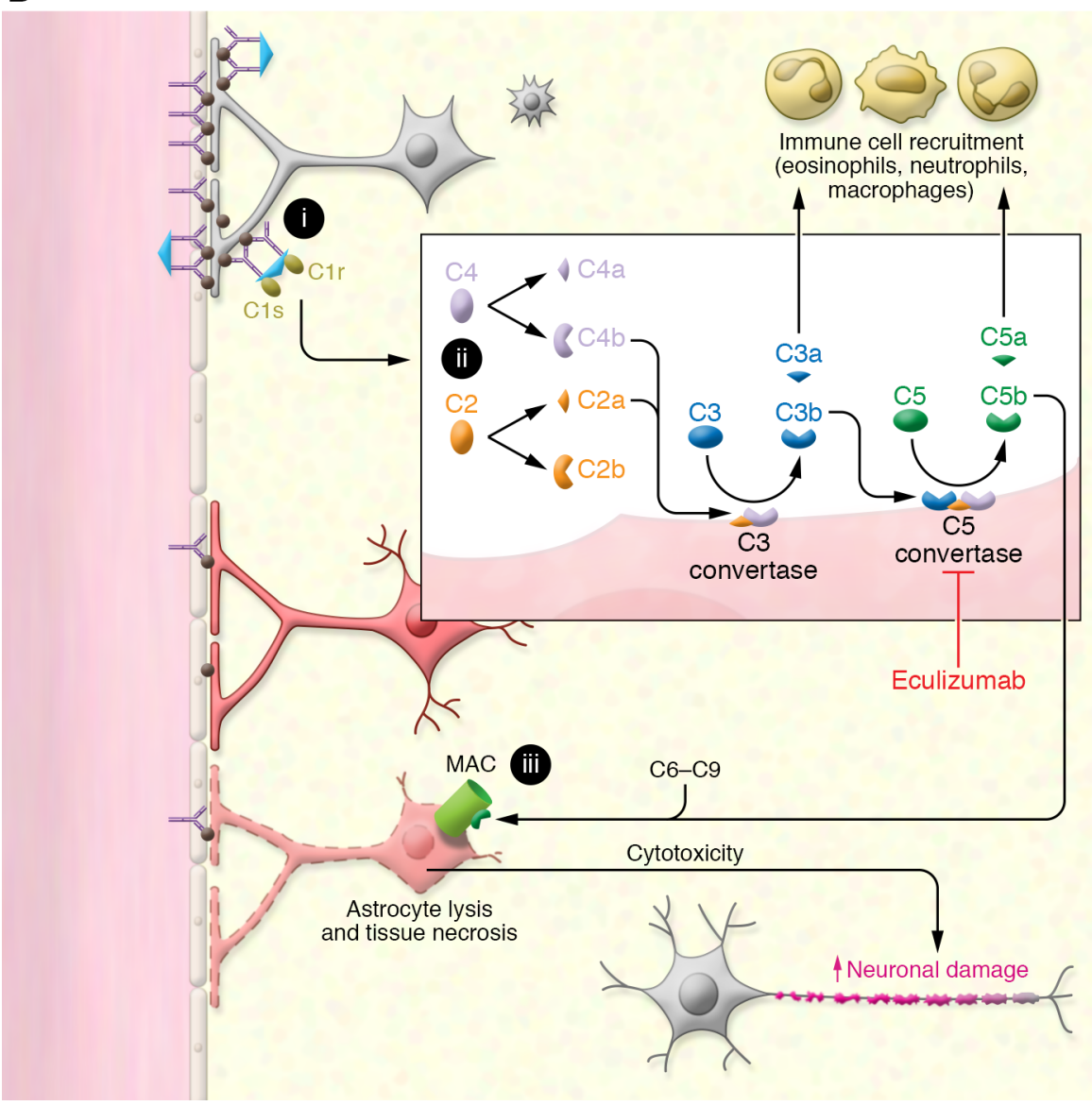

confirming that the $\mathrm{C} 3-\mathrm{C} 3 \mathrm{aR}$ axis was necessary for microglial activation, proliferation, and convergence toward astrocytes, as well as motor impairment.

\section{C1q produced by activated microglia may promote neuronal dysfunction}

The role of the classical complement pathway in NMO pathogenesis is well established $(4,5,9)$. C1q, which is the first protein of the classical complement cascade, becomes engaged when $\mathrm{AQP} 4$-specific IgG is bound to AQP4 on astrocytes and leads to downstream activation of the terminal membrane attack complex (MAC), astrocyte lysis, and tissue necrosis (Figure 1B). Administration of $\mathrm{C} 1 \mathrm{q}$ inhibitors in mouse models of NMO protected astrocytes, oligodendrocytes, and neurons from damage (10). The importance of the complement pathway in NMO is underscored by the recent approval of the C5 inhibitor, eculizumab (Soliris), in treatment of AQP4-seropositive NMO (11).

The work by Chen et al. highlights 2 features of AQP4 antibody- and complement- 
mediated NMO pathogenesis. First, they demonstrated that binding of NMO-IgG to $\mathrm{AQP} 4$ on astrocytes in vivo causes a precytolytic phase that is characterized by astrocytic activation and $\mathrm{AQP} 4$ reduction in the absence of terminal complement activation (6). Mice were well suited for their study, as it is well known that this species produces endogenous inhibitors that limit terminal activation of the classical complement pathway (12). Second, the Chen et al. study established a unique role for C3 in astrocyte-microglia crosstalk in the early phase of AQP4 antibody-mediated CNS autoimmunity that led to microglial activation (6). Previously, it was observed that proinflammatory (M1) microglia promote development of reactive astrocytes (13). Now, showing that astrocytic C3 production activates microglia emphasizes a bidirectional relationship between these 2 cells in CNS inflammation.

Microglia are a principal CNS source of C1q and evidence indicates that C1q alone can promote axonal damage in neurodegenerative disease (refs. 14, 15, and Figure 1A). Thus, C1q may have a dual role in CNS injury in NMO, initiating the classical complement cascade and, separately, participating as a toxic mediator of neuroglial damage. Although Chen and colleagues demonstrated that intrathecal NMO-IgG administration promoted activation of microglia and increased C1q expression by microglia (6), additional studies are needed to confirm its physiologic relevance to pathogenesis. Was paralysis that was induced by NMO-IgG dependent on $\mathrm{Clq}$ produced by microglia? In further investigation, it may be important to learn if eliminating $\mathrm{C} 1 \mathrm{q}$ production by microglia alone prevents motor symptoms and reduces tissue injury induced by NMO-IgG.

\section{Microglia may be a target for NMO therapy}

NMO has an "outside-in" pathophysiology (16). In this regard, the adaptive immune response (i.e., generation of anti-AQP4 antibodies) that drives NMO occurs outside the CNS. New therapies being considered for approval, e.g., B cell depletion (17) and anti-IL-6 (18), primarily target peripheral aspects of NMO immunopathogenesis. Yet, it is the consequent participation of innate immunity within the CNS that is responsible for tissue destruction. Comple- ment activation occurs in situ and is generally considered responsible for recruitment of neutrophils and eosinophils, the 2 leukocytes characteristically identified in NMO lesions (4). Microglia, which have been largely neglected in NMO, are also abundant. The report by Chen, et al. now draws attention to the potential cooperation of astrocytes and microglia in NMO (6). Their new insights are relevant to AQP4-seropositive patients, who account for $75 \%$ of individuals with an NMO phenotype $(19,20)$. Whether microglia can serve as a new target in NMO, as suggested by the authors, is uncertain. Currently, targeting microglia has been challenging. Many microglial markers are not entirely selective and may be expressed to some extent by peripheral myeloid cells, e.g., macrophages and dendritic cells $(21,22)$. Identification of targetable microgliaspecific gene products is actively being pursued, and may be relevant, not only to NMO, but other inflammatory and neurodegenerative CNS diseases associated with microglial activation. Regardless, the observations made by Chen and colleagues (6) should encourage investigators to further study astrocyte-microglia crosstalk in CNS diseases and the role of microglia in NMO.

\section{Acknowledgments}

We thank Joseph J. Sabatino and Patricia A. Nelson for helpful discussion and careful review of the manuscript. ZM is a postdoctoral research fellow of the National Multiple Sclerosis Society (NMSS) (FG1708-28893). SSZ receives research grant support from the NIH (R01 AI13162401A1 and R21 NS 108159-01), NMSS (RG1701-26628), Weill Institute, Race to Erase MS, and the Maisin Foundation.

Address correspondence to: Scott S. Zamvil, Department of Neurology, UCSF, 675 Nelson Rising Lane, NS-215A, San Francisco, California 94158, USA. Phone: 650.799.2101; Email: zamvil@ucsf. neuroimmunol.org.

1. Lennon VA, et al. A serum autoantibody marker of neuromyelitis optica: distinction from multiple sclerosis. Lancet. 2004;364(9451):2106-2112.

2. Lennon VA, Kryzer TJ, Pittock SJ, Verkman AS, Hinson SR. IgG marker of optic-spinal multiple sclerosis binds to the aquaporin- 4 water channel. JExp Med. 2005;202(4):473-477.
3. Varrin-Doyer M, et al. Aquaporin 4-specific T cells in neuromyelitis optica exhibit a Th17 bias and recognize Clostridium $\mathrm{ABC}$ transporter. Ann Neurol. 2012;72(1):53-64.

4. Lucchinetti CF, et al. A role for humoral mechanisms in the pathogenesis of Devic's neuromyelitis optica. Brain. 2002;125(pt 7):1450-1461.

5. Lucchinetti CF, Guo Y, Popescu BF, Fujihara K, Itoyama Y, Misu T. The pathology of an autoimmune astrocytopathy: lessons learned from neuromyelitis optica. Brain Pathol. 2014;24(1):83-97.

6. Chen C, et al. Astrocyte-microglia interaction drives evolving neuromyelitis optica lesion. J Clin Invest. 2020;130(8):4025-4038.

7. Howe CL, Kaptzan T, Magaña SM, Ayers-Ringler JR, LaFrance-Corey RG, Lucchinetti CF. Neuromyelitis optica IgG stimulates an immunological response in rat astrocyte cultures. Glia. 2014;62(5):692-708.

8. Davoust N, Jones J, Stahel PF, Ames RS, Barnum SR. Receptor for the C3a anaphylatoxin is expressed by neurons and glial cells. Glia. 1999;26(3):201-211.

9. Soltys J, et al. Membrane assembly of aquaporin-4 autoantibodies regulates classical complement activation in neuromyelitis optica. J Clin Invest. 2019;129(5):2000-2013.

10. Phuan PW, et al. C1q-targeted monoclonal antibody prevents complement-dependent cytotoxicity and neuropathology in in vitro and mouse models of neuromyelitis optica. Acta Neuropathol. 2013;125(6):829-840.

11. Pittock SJ, et al. Eculizumab in aquaporin-4 positive neuromyelitis optica spectrum disorder. N Engl JMed. 2019;381(7):614-625.

12. Ratelade J, Verkman AS. Inhibitor(s) of the classical complement pathway in mouse serum limit the utility of mice as experimental models of neuromyelitis optica. Mol Immunol. 2014;62(1):104-113.

13. Liddelow SA, et al. Neurotoxic reactive astrocytes are induced by activated microglia. Nature. 2017;541(7638):481-487.

14. Dejanovic B, et al. Changes in the synaptic proteome in tauopathy and rescue of tauinduced synapse loss by C1q antibodies. Neuron. 2018;100(6):1322-1336.e7.

15. Györffy BA, et al. Local apoptotic-like mechanisms underlie complement-mediated synaptic pruning. Proc Natl Acad Sci US A 2018;115(24):6303-6308.

16. Sabatino JJ, Pröbstel AK, Zamvil SS. B cells in autoimmune and neurodegenerative central nervous system diseases. Nat Rev Neurosci. 2019;20(12):728-745.

17. Cree BAC, et al. Inebilizumab for the treatment of neuromyelitis optica spectrum disorder (N-MOmentum): a double-blind, randomised placebo-controlled phase 2/3 trial. Lancet. 2019;394(10206):1352-1363.

18. Yamamura T, et al. Trial of satralizumab in neuromyelitis optica spectrum disorder. $\mathrm{N}$ Engl J Med. 2019;381(22):2114-2124.

19. Wingerchuk DM, Lennon VA, Lucchinetti CF, Pittock SJ, Weinshenker BG. The spectrum of neuromyelitis optica. Lancet Neurol. 2007;6(9):805-815.

20. Zamvil SS, Slavin AJ. Does MOG Ig-positive 
AQP4-seronegative opticospinal inflammatory disease justify a diagnosis of NMO spectrum disorder? Neurol Neuroimmunol Neuroinflamm. 2015;2(1):e62.
21. Butovsky $\mathrm{O}$, et al. Identification of a unique TGF- $\beta$-dependent molecular and functional signature in microglia. Nat Neurosci. 2014;17(1):131-143.
22. Ajami B, et al. Single-cell mass cytometry reveals distinct populations of brain myeloid cells in mouse neuroinflammation and neurodegeneration models. Nat Neurosci. 2018;21(4):541-551. 